\title{
Optical non-linearities due to charge transfer in type II heterostructures
}

\author{
R. PLANEL, R. TEISSIER and F. MOLLOT \\ Laboratoire de Microstructures et Microélectronique, Centre National de la Recherche Scientifique \\ (L2M-CNRS), BP. 107, 92225 Bagneux cedex, France
}

\begin{abstract}
We present experimental studies of photocreated carrier population in type II heterostructures. The main effects are related to charge separation and band filling : Blue shift and broadening of the luminescence line, transition from indirect recombination to pseudo-direct, and then to direct-type 1 recombination. They may lead to more complex non-linear effects such as bistability. These effects can be accounted for by theory in the framework of the Envelope-Function Approximation, by self consistent treatment of the Poisson and Schroedinger equations.
\end{abstract}

\section{INTRODUCTION}

Type II Heterostructures has been the subject of much less investigations than the more classical, type I systems (For a review, see ref. 1). The GaAs/AlAs system was evidenced to be of type II in $1986(2,3)$. It has the unique advantage to be continuously compatible with the canonical type system based on the same family of host materials. Due to the small lattice mismatch between $\mathrm{GaAs}$ and $\mathrm{AlAs}, \mathrm{Ga}_{1-x} \mathrm{Al}_{x} \mathrm{As}$ alloys with any $\mathrm{Al}$ concentration $x$ may be epitaxially grown on the same layers, with excellent, actually the best, control of thicknesses and concentrations. As a matter of facts, several structures have been recently proposed which blend type I and type II properties $(4,5)$. Thus, the epitaxial grower may create a wide variety of structures, having in mind to engineer various peculiar properties. The most obvious idea is to make capital out of the spatial separation of carriers in different layers of the structure to create electric fields, which enhance the non-linear optical properties of the structure. In addition to the interest of engineering, such effects are attainable with low power light excitation, due to the slow recombination rates of spatially separated carriers. As far as potential applications to devices are concerned, this advantage is counterbalanced by slow recovery when excitation is switched off, illustrating once more a general limitation in device physics.

This paper is organized as follows. In Section 2, we recall the basic features of electronic states of type II structures, on the basis of experimental results obtained in GaAs/AIAs short period superlattices. In Section 3, we present an experimental study of charge carrier build-up in "type II quantum wells", the simplest structures to evidence clear experimental results under high excitation. It is a must to understand some optical nonlinearities which are presented in Section 4. 


\section{BAND STRUCTURE AND ELECTRONIC STATES OF TYPE II HETEROSTRUCTURES}

\subsection{Type I versus Type II heterostructures}

Superlattices (SL) made of GaAs and AIAs were first investigated by several groups $(2,3,6)$. Actually, the ability to get type II structures is closely linked to the band offset between the two host materials. The key feature is the following : the $X$ conduction band extremum of AlAs bulk material lies at lower energy than the equivalent extremum in GaAs. This allows to assert that heterostructures built in this system may be of type II, as far as X-potential profiles are concerned, since the situation of valence states is not, in GaAs/AlAs heterostructures, substantially different from the case of GaAs/GaAlAs. This happens either when indirect materials are used (i.e. with $x>0.35$, typically), or when confinement effects rise the $\Gamma$ states at high enough energy for the $X$-states to become the conduction fundamental state. Systematic experimental studies of GaAs/AlAs SL have been done, mainly by mean of $\mathrm{cw}$ photoluminescence (PL) and excitation of luminescence (PLE) spectroscopies (3). The conclusion of such studies may be drawn as illustrated in Fig 1 . Roughly speaking, the GaAs/AlAs superlattice is "direct-type I" if the GaAs layer thickness $L_{G a A s}$ is larger than 3.5 to $4 \mathrm{~nm}$ or if the average Aluminium concentration $x$ (defined as

$L_{\text {AlAs }} /\left(L_{\text {GaAs }}+L_{\text {AIAs }}\right)$ is smaller than 0.35 , the critical concentration for direct to indirect gap transition in GaAlAs alloys ( these numerical values are little dependant on the authors, but stightly on temperature, since the $X$ and $\Gamma$ temperature coefficient are very different). The second criterion is of course more efficient for very short period SL's, which may be considered as "pseudo-alloys". If neither of these criterion is met, one gets a type II superlattice, with electrons mainly located in the AlAs layers, and holes remaining in the GaAs layers.

\subsection{Indirect versus pseudo-direct recombination}

For a more accurate understanding of the $X$-conduction state, it has been necessary to rely on time resolved luminescence (7) and uniaxial strain perturbation (8). The features are somehow intricate because of the small lattice mismatch between the bulk materials GaAs and AlAs, which results in a biaxial strain of AlAs to match the lattice constant fixed by the GaAs substrate. This lowers the energy of the $X_{x, y}$ extrema of the AlAs band structure ( which are situated in k-direction 100 and 010 , within the plane of the layers ), with respect to the $X_{z}$ one (which lies along 001 , the growth direction). But the $X$-valleys are highly anisotropic, with cigar-like constant energy surfaces. As a consequence, the confinement effect rises the $X_{x, y}$ energy in a more pronounced way than the $X_{z}$ energy. It 
is the balance between confinement and strain effect which will determine the symmetry of the fundamental $X$-states. In an heterostructure, the $X_{z}$ states do not retain a periodic term in their envelope-function, due to the folding of the Brillouin zone. Experimentally, it expresses very clearly into an intense zero -phonon recombination appearing on the photoluminescence spectra, and a more rapid decay (typically $1 \mu \mathrm{s}$ ) than for $X_{x, y}$ states (100us to $1 \mathrm{~ms}$ ) (7). The $x_{z}$ recombination can be said pseudo-direct, since direct recombination, without phonon emission, is allowed by the heterostructure potential, although the system remains of type II (sometimes said "indirect in real space").

Thus, it has been possible to determine the both regions into the $L_{A I A s} v s . L_{\text {GaAs }}$ map of Fig. 1. The SL. fundamental conduction states are $X_{x, y}$ like if $L_{A I A s}>7 n m$, or for very thin $L_{\text {GaAs, }}$ typically $<1 \mathrm{~nm}$.

\subsection{The Envelope Function Approximation : an essential tool for modelization}

A major conclusion of these studies is the outstanding efficiency of theories based on the envelope function approximation (9). Despite the small dimensions (a few $\mathrm{nm}$ ) of layers, which make its fundation uncertain, this type of calculation reveals in very good agreement with experimental results, explains their variation with the heterostructure parameters, and allows predictive conclusions. It is an essential tool for engineering, especially when treated by a transfer matrix method (10). Then, any kind of potential shape may be considered, including the effect of electric field, carrier population through Poisson equation, etc...

\section{STUDY OF CHARGE BUILD-UP IN TYPE II QUANTUM WELLS}

\subsection{Experimental conditions}

As far as high optical excitation is concerned, the problem of homogenous excitation is essential. This is even more dramatic when electric fields are involved, either externally applied or internal. In order to deal with this problem, we have studied oversimplified structures, which may be named "type II quantum wells". It consists of a single bi-layer of GaAs/AlAs, flanked by wide, typically $100 \mathrm{~nm}, \mathrm{Ga}_{0.65} \mathrm{Al}_{0.35} \mathrm{As}$ barriers. Let us point out that this alloy is confining all kinds of electrons in the central part of the structure : $\mathbf{r}$ as well as $X$ electrons (see Fig 2). Since there is only one cell into the structure of interest, the homogeneity along the z-direction of the carrier population is optimized. The lateral homogeneity, that is in the plane of the layer, has also to be controled : we used moderately focused laser excitation $(100 \mu)$ and made a spatial filtration of the spot image to restrict photon acquisition to its central part.

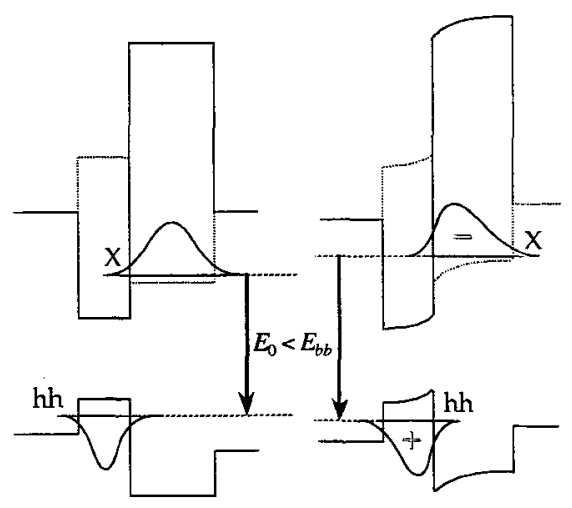

Figure 2 : Sketch of the band bending due to carrier of the spatially separated layers. $E_{O}$ is the band gap energy without illumination. $E_{b b}$ is the blue-shifted band gap energy. 
Such structures have been grown by Molecular Beam Epitaxy, and are often inserted as the intrinsic part of $p-i-n$ or $n-i-n$ diodes. As a matter of fact, an other point to be taken into account for precise measurements and comparisons in type II systems, is the electric field intensity and sign in the absence of optical excitation ; this requires a reasonnable control of the doping of the substrate, buffer and cap layers. The estimation of the residual doping of the intrinsic parts is more difficult, as well as the effect of carrier transport perpendicular to the layers. To that respect, as we shall see later, working on well defined mesa-type structures may be of great interest to compare open-circuit and short-circuit behaviour, as well as to apply electric fields. It is more difficult to get reproducible results in as-grown layers, because of hazardous short circuits induced by defects. In the following sub-sections, we show that it is possible to get a very good control and understanding of the population of type II, either pseudo-direct or indirect, quantum wells.

For that purpose, we present results on some representative structures. We have mainly used $\mathrm{CW} \mathrm{PL}$ and PLE measurements at low temperature $(T=2 \mathrm{~K})$. In some cases timeresolved PL. measurements also bring interesting information.

\subsection{Experimental Results}

We shall now present results obtained in a single GaAs $(2.5 \mathrm{~nm} / /$ AlAs $(5 \mathrm{~nm})$ bi-layer, surrounded by $100 \mathrm{~nm}$ thick $\mathrm{Ga}_{0.65} \mathrm{Al}_{0.35} \mathrm{As}$ barriers on each sides. From calculation, it is predicted to be pseudo-direct, that is with a $X_{2}$ symmetry conduction ground state, because the AlAs layer is thinner than $7 \mathrm{~nm}$. This structure forms the intrinsic part of $p-i-n$ diode or $n-i-p$ diode. In the former (resp. the latter), the built-in electric field of the diode rises the energy of the GaAs (resp. AlAs) layer with respect to its AlAs (resp.GaAs) neighbour.

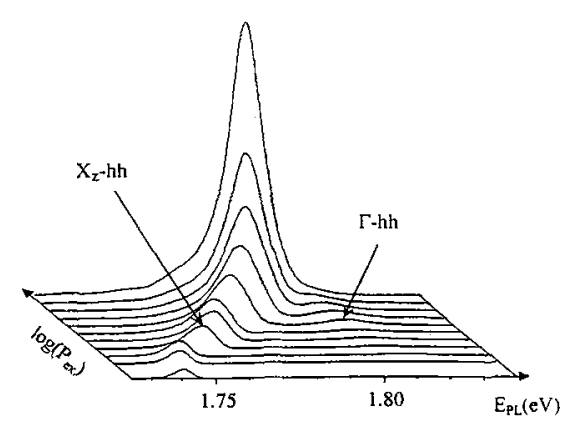

Figure 3 : Luminescence spectra of a pseudo-direct GaAs (2.5nm)/AlAs (5nm) type II quantum well at various pump intensities.
At low level excitation, typically a few $\mathrm{mW} / \mathrm{cm}^{2}$, the luminescence spectra of such structures are quite similar to the one of pseudo-direct type II superlattices : one main, intense no-phonon line associated to three smaller phonon replicas. The r-HH direct recombination into the GaAs well is also observed, as a weak line. In a first step, care is taken to use an exciting energy smaller than the barrier height, in order to create carriers inside the type II well, and to ensure equal population of electrons and holes in the heart of the structure. As the exciting power is increased, up to $100 \mathrm{~W} / \mathrm{cm}^{-2}$, the line shifts towards high energies (blue shift) by some $60 \mathrm{meV}$ in the $\mathrm{p}-\mathrm{i}-\mathrm{n}$ structure, and widens (see Fig. 3 ). In the same time the $\mathbf{r}$ $\mathrm{HH}$ line is slightly red-shifted (10meV). When these two lines merge into one, the energy shift saturates, and the line becomes narrower. During this evolution, the integrated intensity increases close to linearly with the exciting power, and it is

mainly due to the increase of the linewidth. In other words, we fill the electron and hole subbands, with a constant two-dimensionnal density of states. We now show that we can describe quantitatively this effect. 


\subsection{Modelization}

The blue shift is first a consequence of the band bending induced by charge accumulation into the bilayer. Roughly speaking, the spatial separation of electron and holes creates an electric field which risens the transition energy (see Fig. 2). We can calculate it by a self consistent treatment of the Poisson equation and of the Schroedinger equation for the envelope functions of electrons and holes. As we shall see, it is sufficient to consider one populated subband for each type of carrier. We also have to assume, for example, that they are equally populated ; this is based on our exciting energy conditions. Thus, we get the band bending and the transition energy for a given value $n$ of the density. It appears that for sheet densities smaller than $10^{12} \mathrm{~cm}^{-2}$, the energy shift of the transition is close to linear with $n$. For our structure parameters, we find $\Delta E_{0}=4.0 \mathrm{n}$, where $\Delta \mathrm{E}_{0}$ should be close to the low energy side of the line. It is expressed in meV and $n$ in $10^{11} \mathrm{~cm}^{-2}$.

To describe the line widening, we first estimate the relation between the quasi Fermi level $\mu$ of each subband and the density : $\mu=\pi n \hbar 2 / \mathrm{m}$ where $\mathrm{m}$ is the transverse effective mass of the particle of interest. For $X_{z}$ electrons, we can use the bulk AlAs value for the light mass of $X$ states, since electrons are essentially confined in AlAs layers : $m_{X z}=0.19$, which leads to $\mu^{X z}=1.26 \mathrm{n}$. For $X_{X, Y}$ electrons, this analysis also holds, although it is not of interest in the present case ; there, we have to use for the transverse density of states mass the geometrical average of $X$-states light and heavy masses, and to account for the two equivalent valleys: $m_{X x, y}=(1.1 \times 0.19)^{1 / 2}$, which leads to $\mu^{X x, y}=0.26 \mathrm{n}$. For holes, the situation is more complicated, due to the heavy-light coupling. We have calculated the dispersion relation of heavy holes for our structure within the framework of the Luttinger description (9), and found $m_{h h}=0.11$, which leads to $\mu^{\text {hh }}=2.18 n$.

To get the linewidth from the quasi-Fermi levels, we need some more details on the recombination process. In bidimensionnal heterostructures, non-vertical recombinations due to interface roughness are often invoked to explain the experimental results. In our case, with high densities, the question is even more obscure. As a consequence, we shall consider the two extreme models, either k-conserving or $k$-independant recombination. The theoretical width of the line is different in each case, as shown in Fig. 4. Here, we have taken into account the fact that the Fermi energy is larger for holes than for $X$ electrons. With the numerical values obtained above, we can express the theoretical expression for the linewidth in both cases : in the $k$ conserving case $\Delta W=3.44 n$; in the $k$ independant case, $\Delta W=2.18 \mathrm{n}$. We now consider the energy shift of the line, measured at maximum, to be due to the band bending and to the Fermienergy rising, which are both linearly
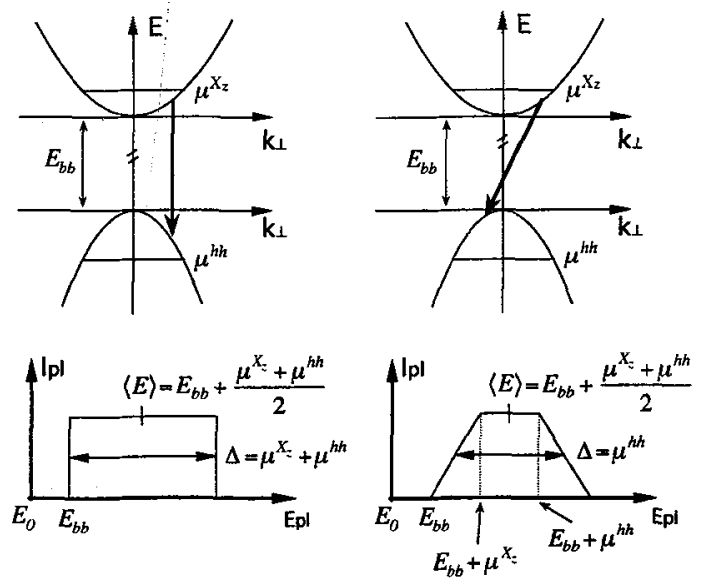

Figure 4 : Theoretical lineshape of radiative transitions with (left) or without (right) conservation of the in-plane wavevector. $\mu$ are the Femi energies relevant for each type of particle ; $\langle E\rangle$ is the mean energy of the line : $\Delta$ is the half maximum width. 
dependant with the sheet density :

$\Delta E=\Delta E_{0}+\Delta W / 2$.

Thus, we infer that the ratio of the shift to the widening should be constant : to summarize, in our structure, we expect :

in the $k$-conserving case : $\Delta E / \Delta W=1.66$

in the $k$-independant case $: \Delta E / \Delta W=2.33$

In Fig 5, we have plotted, for several exciting powers, the experimental blue shift as a function of the measured linewidening (1). This simple approach accounts for the results, at lowest densities. It allows to select a model for recombination, showing that the $k$--selection rule remains valid in our structure. And we may extract from experimental data the sheet density with a good level of confidence. High sheet densities, up to $710^{11} \mathrm{~cm}^{-2}$, are obtained with reasonnable excitation power, typically $100 \mathrm{~W} / \mathrm{cm}^{-2}$. It corresponds to $\mu^{\mathrm{hh}}=15 \mathrm{meV}$, and $\mu^{X z}=9 \mathrm{meV}$. Thus, we can also check that other subbands do not interfere with the population of the fundamental ones in our case : neither light holes (situated some $50 \mathrm{meV}$ above the heavy holes), nor the $X_{x, y}$ states (15 meV above the $X_{z}$ states), nor the $X_{z} 2$ states (30meV above).

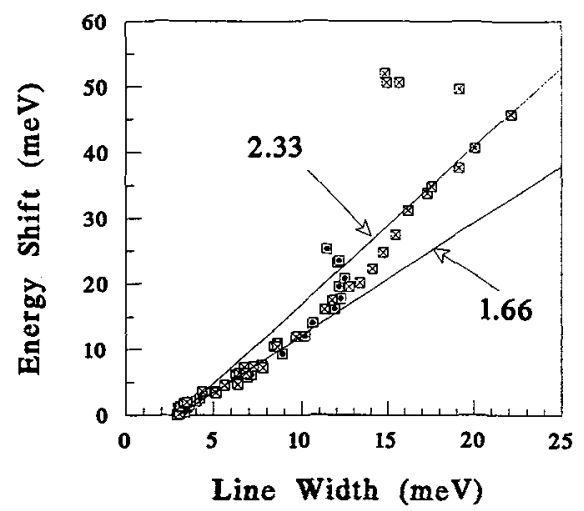

Figure 5 : Energy shift versus linewidth of the pseudodirect luminescence of a GaAs (2.5nm)/AIAs (5nm) type II quantum well, which forms the intrinsic part of a $p-i-n(x)$ or $n-i-p(\bullet)$ diode.

\section{OPTICAL NON-LINEARITIES}

\subsection{Type II to Type I transition}

On the opposite, the $\boldsymbol{\Gamma}$ - recombination comes into play, as we have seen in Fig.3, acting as an overflow recombination for $\mathrm{X}$-electrons. As a matter of fact, it expresses by a drastic reduction of the luminescence decay time (from the $\mu$ s range to the ns range). On Fig 5, our simple model breaks down earlier, probably due to the leakage of charges in the barriers. The $\Gamma$ to $X$ distance in such a structure is about $50 \mathrm{meV}$, in zero electric field. But this quantity is dramatically dependant on the photocreated field, because $\mathbf{r}$ and $X$ states are not essentially localized in the same layers. Thus the $\Delta E$ E quantity has to be substracted to the initial $\Gamma-X$ splitting, and the $\Gamma$-level can be reached by the $X_{z}$ electron Fermi-level. Actually, the built-in field has to be taken into account, as is evidenced by the difference between the $p-i-n$ and $n-i-p$ structures. In the former case, the photocreated field opposes to the built-in field, allowing a better filling of the $X$ states. This effect is in facts related to the Type I to Type II transition induced by an external field effect, which has also been evidenced (11).

(1) note: To get the experimental widening of the line, we extract the "natural' width, most probably due to heterogenous broadening, which is obtained at low power. In this actual structure, it amounts to $3 \mathrm{meV}$. 
Last, we cannot ignore that the $\mathbf{\Gamma}-\mathrm{X}$ splitting is further reduced by the renormalisation of the direct band gap, which hardly appears (10meV) in our conditions. Actually little is known on this effect on the type II transitions. This could decrease the $X$-transition energy. However, the good agreement between our results and our simple model indicates that such effects can be neglected in our experimental conditions. Moreover, recent studies on Type II SL (12) confirms that the electrostatic and Fermi terms are prevailing in structures such as ours.

\subsection{Indirect to pseudo-direct transition}

We shall now present results obtained on a indirect type II quantum well, that is with a $X_{x, y}$ fondamental conduction level ; however, we choosed a structure close to the indirect to pseudodirect transition, that is with a small splitting with the (upper) $X_{z}$ level, to evidence the progressive population of the $X_{z}$ level. It consists in one bilayer : GaAs $(2.5 \mathrm{~nm})$ /AIAs $(8 \mathrm{~nm})$, imbedded into a wide $(100 \mathrm{~nm})$ GaAlAs barrier. From theory, the $X_{x, y}-X_{z}$ splitting can be estimated to be $2 \mathrm{meV}$, with of course a large relative incertainty.

Due to the higher radiative yield of pseudodirect transitions, it is possible to observe lower charge densities in the $X_{z}$ level. As a matter of facts, as excitation power is increased, we observe experimentally a continuous change from an indirect to a pseudodirect recombination spectrum (see Fig 6) : the zero-phonon line progressively prevails as the spectrum shifts towards high energies. This blue shift tends to inflect as the $X_{2}$ level is populated (see Fig 7 ), which reflects the higher recombination rate of charge built-up in the $X_{z}$ level. The continuous curve results from a theoretical calculation based on a simple modeling of the band filling : we use the Fermi Dirac statistics of the conduction electrons, rate equations based on the experimental lifetimes of the luminescence, and the selfconsistent treatment of the Poisson and Schroedinger equations for the envelope functions of electrons and holes. Details will be published elsewhere (13), but we wish to point out here the outstanding sensitivity of this fitting to the two unknown parameters : the $X_{x, Y^{-}}$ $X_{z}$ splitting on one hand, the electronic temperature on the other hand. Both are obtained with a $10 \%$ precision, and we may note the remarkable thermalization of the carriers with the lattice temperature. This is a consequence of the long lifetimes in these type II structures.

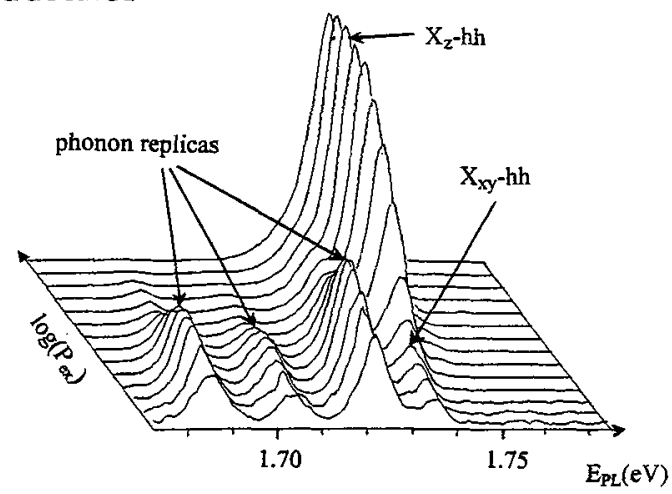

Figure 6: Luminescence spectra of an indirect GaAs (2.5nm)/ AlAs (8nm) type II quantum well at various pump intensities.

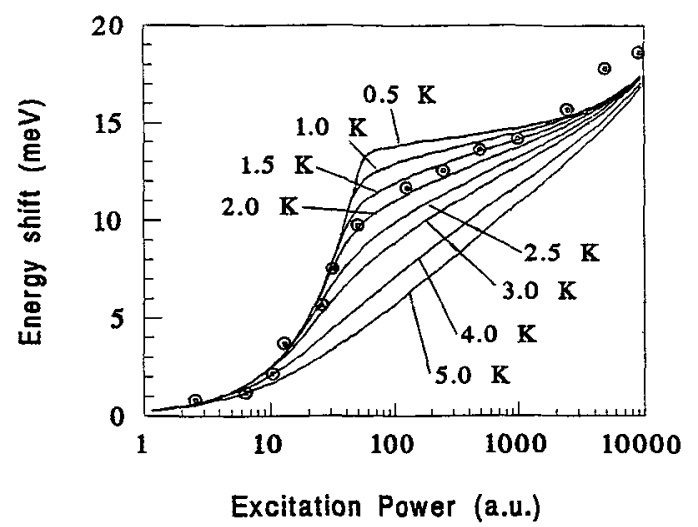

Figure 7 : Fit of the experimental (dots) energy shift as a function of pump intensity, for various temperatures. 


\subsection{Optical bistability}

Although these results are established in very specific structures and experimental conditions, they are of great interest to understand the behaviour of more complicated ones. For example, we have studied an another indirect type II quantum well, with larger $x_{x, y^{-}} X_{z}$ splitting, (GaAs $2.5 n m$ /AIAs 10nm), inserted into a $n$-i-n diode, and under excitation above the barrier energy. Then, charges may accumulate in other places than the type II quantum well itself. Actually, we have been able to follow the progressive population of first $X_{x, y}$, then $X_{z}$ electrons, up to the point where they merge into the $r$ level. It is at this point that this structure, exhibit optical bistability, as presented in details elsewhere (14). The barrier luminescence intensity is increased between the two stable states by one or two orders of magnitude. This reflects a rapid change of the charge repartition in the whole structure. This unstabilization is due to transition from a slow type II to a rapid type I recombination in the heart of the structure, the type II quantum well. The positive feed-back which stabilizes the strong luminescence state is provided by an internal electric field, due to the new charge repartition, which rises the $X$ state energy with respect to the $\Gamma$ one.

\section{CONCLUSION}

We have shown that the behaviour of type II heterostructures may be reasonnably mastered, and used for engineering. Optical non-linearities appear for very low exciting power, at least at low temperature. Inclusion of such active structures into Jarger devices, such as optical microcavities, may be an interesting field for future works.

\section{References}

[1] Wilson B.A., IEEE J.Quantum Electron. QE-24 (1988) 1763

[2] Finkman E., Sturge M. D. , and Tamargo M. C. , Appl. Phys. Lett. 49, (1986)1299

[3] Danan G. et al. Proc.18th Internat.Conf.Phys.Semicond., Stockholm 1986, ed.O.Engström, World Scientific, Singapore 1987, p. 719 ; Phys. Rev. B 35, (1987) 6207 [4] Jezewski M. , Teissier R. , Mollot F. and Planel R. , Superlattices and Microstructures, Vol. 8, No. 3, (1990) 329

[5] Galbraith I., Dawson P. and Foxon C.T., Phys.Rev B45 (1992) 13499

[6] Moore K. J. , Dawson P. , and Foxon C. T. , Journal de Physique (Paris) C5, (1987) 525 ; Phys. Rev. B 38, (1988) 3368

[7] Scalbert D. et al., Solid State Com., 70, 945, (1989); Maaref M. et al. Phys.Stat.Sol., 170 (1992) 637

[8] Lefebvre P., Gil B., Mathieu H. and Planel R., Phys Rev. B39 (1989) 5550 ; Phys.Rev. B40 (1990) 7802

[9] Bastard G. Phys. Rev. B, 24, (1981) 5693; Wave mechanics applied to semiconductor heterostructures, (Les éditions de physique, Les Ulis 91-France ,1988).

[10] Yuh Peng-Fei and Wang K.L., Phys.Rev. B38 (1988) 13307

[11] Meynadier M.H. et al. Phys. Rev. Lett. 60 (1988) 1338.

[12] Boujdaria K. Thèse de l'Universite Paris 7, France (1993) ; to be published

[13] Teissier R. et al., to be published

[14] Teissier R., Planel R., and Mollot F., Appl.Phys.Lett. 60 (1992) 2663 